\title{
Diminished appetitive startle modulation following targeted inhibition of prefrontal
}

SUBJECT AREAS:

PREFRONTAL CORTEX

DEPRESSION

Received

17 September 2014

Accepted

10 February 2015

Published

10 March 2015

Correspondence and requests for materials should be addressed to R.H. (renehurlemann@ icloud.com)

\section{cortex}

René Hurlemann ${ }^{1,2}$, Stephan Arndt ${ }^{1,2}$, Thomas E. Schlaepfer ${ }^{1,3}$, Juergen Reul ${ }^{4}$, Wolfgang Maier ${ }^{1,5}$ \& Dirk Scheele ${ }^{1,2}$

'Department of Psychiatry, University of Bonn, 53105 Bonn, Germany, ${ }^{2}$ Division of Medical Psychology, University of Bonn, 53105 Bonn, Germany, ${ }^{3}$ Department of Psychiatry \& Behavioral Medicine, The Johns Hopkins Hospital, Baltimore, Maryland, 21287, USA, ${ }^{4}$ Beta Clinic, 53227 Bonn, Germany, ${ }^{5}$ German Center for Neurodegenerative Diseases (DZNE), 53175 Bonn, Germany.

From an evolutionary perspective the startle eye-blink response forms an integral part of the human avoidance behavioral repertoire and is typically diminished by pleasant emotional states. In major depressive disorder (MDD) appetitive motivation is impaired, evident in a reduced interference of positive emotion with the startle response. Given the pivotal role of frontostriatal neurocircuitry in orchestrating appetitive motivation, we hypothesized that inhibitory transcranial magnetic stimulation (TMS) would reduce appetitive neuromodulation in a manner similar to MDD. Based on a pre-TMS functional MRI (fMRI) experiment we selected the left dorsolateral and dorsomedial prefrontal cortices as target regions for subsequent sham-controlled inhibitory theta-burst TMS (TBS) in 40 healthy male volunteers. Consistent with our hypothesis, between-group comparisons revealed a TBS-induced inhibition of appetitive neuromodulation, manifest in a diminished startle response suppression by hedonic stimuli. Collectively, our results suggest that functional integrity of left dorsolateral and dorsomedial prefrontal cortex is critical for mediating a pleasure-induced down-regulation of avoidance responses which may protect the brain from a depressogenic preponderance of defensive stress.

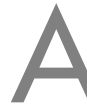

mong the core defensive responses within the human behavioral repertoire is the acoustic startle reflex. The magnitude of this reflex is tightly regulated by aversive and appetitive motivational systems, with the presentation of unpleasant foreground stimuli potentiating and pleasant ones diminishing the startle magnitude, respectively ${ }^{1}$. Consistent with this motivational control of avoidance responses are observations of disrupted appetitive neuromodulation in patients with major depressive disorder (MDD), evident in a reduced interference of positive emotion with the startle response ${ }^{2}$, a noxious preponderance of defensive stress ${ }^{3-5}$, and decreased approach-related behavior ${ }^{6,7}$.

MDD is currently ranked third worldwide in disease burden and is expected to rank first in high-income countries in $2030^{8}$. As many as a third of MDD patients suffer from treatment-refractory depression (TRD). To help those with TRD, a variety of novel brain stimulation techniques have emerged, including transcranial magnetic stimulation (TMS) over the left dorsolateral prefrontal cortex (dlPFC) ${ }^{9}$. Recent meta-analyses have documented response rates of $29.3 \%$ with TMS compared to $10.4 \%$ with sham treatment in $\mathrm{MDD}^{10}$.

Further support for the rationale to apply TMS for modulating dIPFC function comes from studies in healthy individuals. Compelling evidence has accrued that TMS over the dlPFC influences, and interacts with various facets of emotion and motivation. For instance, TMS-induced inhibition of the left dlPFC has been shown to induce behavioral biases towards increased reward responsiveness ${ }^{11-13}$ and enhance both reactive and proactive types of aggression ${ }^{14}$. In contrast, TMS-induced disruption of the right dlPFC diminished subjects' willingness to build a favorable reputation ${ }^{15}$, provoked risk-taking behavior ${ }^{16}$ (but see Ref. 17), and increased the probability of utilitarian moral judgments ${ }^{18}$. While these studies strongly implicate the dlPFC in a top-down regulation of emotion and motivation, less is known about its modulatory impact on mood. Whereas early studies found decreased happiness ratings following excitatory stimulation of the left $\mathrm{dlPFC}^{19,20}$, more recent studies either failed to detect TMS-induced mood changes ${ }^{21,22}$ or measured them only after long-term treatment ${ }^{23}$. On the neural level, excitation of the right dIPFC attenuated right amygdalar responses to negative emotional stimuli in healthy females ${ }^{24}$, adding support to the hypothesis that TMS-induced focal changes in dlPFC activity exert distant modulatory effects within subcortical regions. Consistent with this hypothesis are intriguing findings that 
a

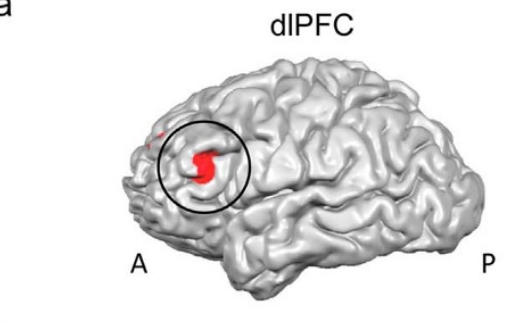

b

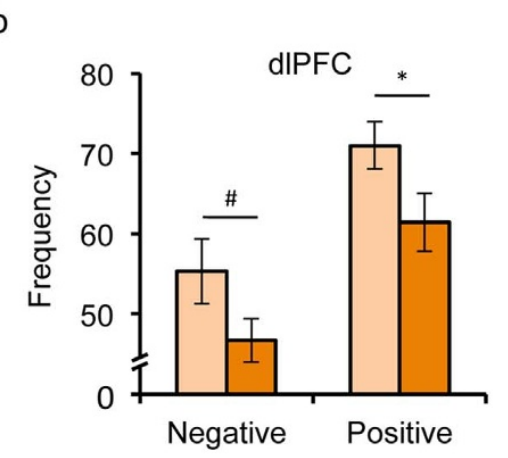

C

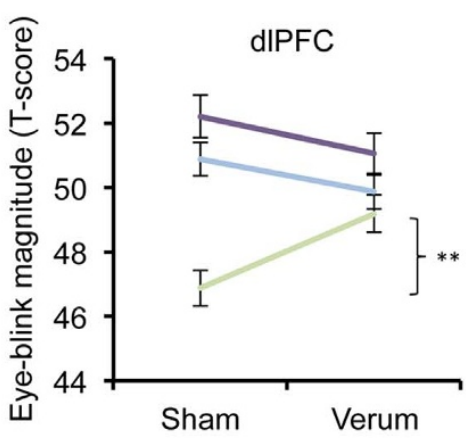

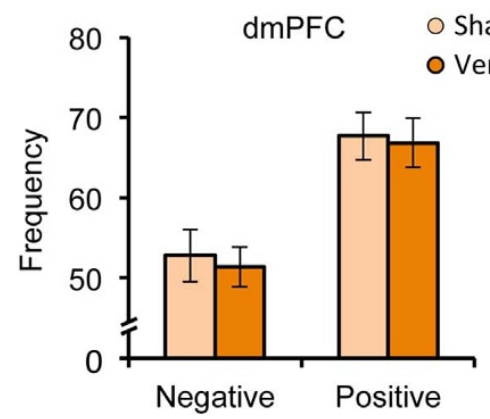

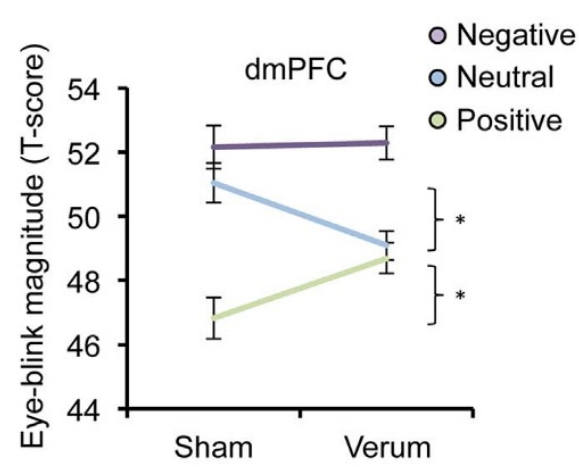

Figure 1 The evaluation of emotional stimuli compared to neutral ones elicited robust responses in the left dorsolateral and dorsomedial prefrontal cortex (dlPFC and dmPFC). The activations are illustrated as $4 \mathrm{~mm}$ spheres around the peak coordinates on the surfaces of the outer grey matter boundary (a). An inhibitory theta burst stimulation (TBS) of the left dlPFC but not of the left dmPFC reduced the frequency of emotion-startle interactions, underscoring the focality of our fMRI-guided intervention. This frequency was defined as the percentage of trials entailing a larger startle magnitude when negative stimuli were presented and a smaller magnitude when positive stimuli were shown (relative to the neutral category) (b). Disruption of the left dlPFC or dmPFC reduced the magnitude of appetitive startle modulation, while leaving a startle potentiation by negative emotion unaffected (c). Error bars indicate the standard error of the mean (SEM). Abbreviations: A, anterior; dlPFC, dorsolateral prefrontal cortex; dmPFC, dorsomedial prefrontal cortex; L, left; $\mathrm{P}$, posterior; $\mathrm{R}$, right; ${ }^{* *} P<0.01 ;{ }^{*} P<0.05 ;{ }^{*} P<0.10$.

TMS over different prefrontal areas alters striatal dopamine (DA) release $^{25-27}$, which is known to substantially contribute to appetitive motivational processes ${ }^{28}$.

Left dlPFC metabolic hypoactivity has been linked to anhedonia in patients with $\mathrm{MDD}^{29}$ and thus supports the therapeutic rationale for using excitatory TMS over this region. Less is known, however, about the mechanistic role of the left dlPFC in appetitive neuromodulation. To address this question, the present study was designed to model a deficit in pleasure-induced startle response suppression in healthy subjects by compromising left dlPFC function with inhibitory TMS. Based on the results of a pre-TMS functional MRI (fMRI) localizer task we selected the left dlPFC and an adjacent area, the dorsomedial prefrontal cortex (dmPFC), as target regions for subsequent shamcontrolled inhibitory theta-burst TMS (TBS) in 40 healthy male volunteers. Our strategy was to use a prolonged intermittent TBS protocol known to produce long-lasting inhibitory after-effects ${ }^{30}$, before subjects were tested on the emotional startle paradigm and a complementary cognitive emotion judgment task. Given the pivotal role of frontostriatal neurocircuitry in orchestrating top-down influences on value assignment to hedonic stimuli ${ }^{31}$, we hypothesized that TBS-induced dlPFC dysfunction would particularly affect striatal responses. We therefore predicted that our intervention would specifically interfere with a startle reflex attenuation by positive emotion, thus modeling a lack of appetitive neuromodulation that constitutes a core characteristic of the depressive phenotype.

\section{Results}

Results of the fMRI localizer task. The arousal-based evaluation of emotional relative to neutral stimuli elicited activations in a broad neurocircuitry involving prefrontal and cingulate cortices, limbic areas as well as parietal and occipital regions (cf. Supplemental Table S2). In the present study, we focused on two dorsal prefrontal regions (dlPFC and dmPFC; cf. Fig. 1a) for two main reasons: first, the vast majority of studies examining potential antidepressant TMS effects targeted prefrontal regions ${ }^{9,10}$, and second, the limited depth effects of TMS do not allow to directly probe the functional integrity of subcortical areas.

Results of the cognitive emotion judgment task. A repeated measures analysis of variance (ANOVA) with treatment (sham vs. verum) as between-subject factor, target region (dlPFC vs. dmPFC), category (negative, neutral, positive) and measurement (pre vs. post) as within-subject factors and the valence ratings as dependent variable revealed a main effect of category $\left(F_{(2,76)}=918.77, P<\right.$ 
Table $1 \mid$ Valence and arousal ratings in the dIPFC session

\begin{tabular}{|c|c|c|c|c|}
\hline & Verum $(n=20)$ Mean (SD) & Sham $(n=20)$ Mean (SD) & $t$ & $P$ \\
\hline Negative pre & $2.37(0.52)$ & $2.36(0.69)$ & -0.08 & 0.93 \\
\hline Negative post & $2.40(0.57)$ & $2.45(0.65)$ & 0.24 & 0.81 \\
\hline Neutral post & $5.05(0.24)$ & $5.01(0.42)$ & -0.37 & 0.72 \\
\hline Positive pre & $6.95(0.62)$ & $7.09(0.76)$ & 0.60 & 0.56 \\
\hline Positive post & $6.92(0.58)$ & $6.85(0.75)$ & -0.32 & 0.75 \\
\hline Negative post & $6.15(1.36)$ & $6.23(1.06)$ & 0.21 & 0.84 \\
\hline Neutral pre & $2.95(0.91)$ & $3.03(1.07)$ & 0.23 & 0.82 \\
\hline Neutral post & $2.95(0.92)$ & $3.12(1.19)$ & 0.52 & 0.60 \\
\hline Positive pre & $5.55(0.98)$ & $5.60(1.30)$ & 0.15 & 0.88 \\
\hline Positive post & $5.76(1.02)$ & $5.61(1.41)$ & -0.40 & 0.70 \\
\hline
\end{tabular}

$0.01, \eta^{2}=0.96$ ), but no main or interaction effect of treatment (all Ps $>0.28$; cf. Tables 1 and 2). Likewise, a repeated measures ANOVA with the arousal ratings as dependent variable also yielded a main effect of category $\left(F_{(2,76)}=292.14, P<0.01, \eta^{2}=0.89\right)$, but no main or interaction effect of treatment (all $P$ values $>0.33$ ). Thus, our TBS protocol did not alter arousal or valence ratings obtained during cognitive emotion judgments.

Results of the emotion-modulated startle response task. To examine the baseline startle response and its habituation, we computed a repeated measures ANOVA with the raw startle response magnitudes from the interstimulus intervals as dependent variable and six time intervals (each with three startle probes) and the target region (dlPFC vs. dmPFC) as within-subject factors. While we observed a clear decline of the startle magnitude across time $\left(F_{(2.69,91.44)}=11.83, P<0.01, \eta^{2}=0.26\right.$, cf. Supplemental Figure $\mathrm{S} 1)$, no significant treatment difference or treatment $\mathrm{x}$ time interaction was evident (all Ps $>0.23$ ). In an exploratory analysis, we also $t$-standardized the baseline startle magnitudes to reduce the high inter-individual variance in the startle responses, but we still did not find significant main or interaction effects of the treatment (all Ps $>0.46)$.

A main effect of category $\left(F_{(2,76)}=29.14, P<0.01, \eta^{2}=0.43\right)$ across target regions and treatment demonstrated the expected emotion modulation of the startle response with concomitant presentation of negative or positive stimuli potentiating or diminishing the response, respectively. However, we also found a significant interaction between treatment and category $\left(F_{(2,76)}=6.13, P<0.01, \eta^{2}=\right.$
0.14). A comparison of the effect sizes indicated that the emotion modulation in the verum group $\left(F_{(2,38)}=8.01, P<0.01, \eta^{2}=0.30\right)$ was almost only half as large as in the sham group $\left(F_{(2,38)}=26.01, P\right.$ $\left.<0.01, \eta^{2}=0.58\right)$. Notably, after dlPFC stimulation the frequency of appetitive startle modulation was reduced, evident in a lower percentage of startle responses with a smaller magnitude during the presentation of positive stimuli compared to neutral ones $\left(t_{(38)}=\right.$ $2.07, P=0.046$, Cohen's $d=0.67$, cf. Fig. $1 \mathrm{~b})$. There was also a trend for a decreased impact of negative information (i.e. a reduced frequency of startle responses with a larger magnitude during the presentation of negative stimuli compared to neural ones; $t_{(38)}=1.75, P$ $=0.09$, Cohen's $d=0.57)$. No such effects were evident after dmPFC stimulation (all $P$ values $>0.73$ ). Concerning the eye-blink magnitude ( $T$ scores), post hoc unpaired t-tests revealed that the modulation of the startle magnitude in response to positive emotion was impaired following verum stimulation of both target areas (dlPFC: $t_{(38)}=2.87, P<0.01$, Cohen's $d=0.93$; dmPFC: $t_{(38)}=2.32, P=$ 0.03 , Cohen's $d=0.75$, cf. Fig. 1c). Put differently, the frequency or penetrance of appetitive as well as aversive neuromodulation was diminished after dlPFC stimulation, whereas its intensity was affected after both dlPFC and dmPFC stimulation, resulting in a selective suppression of appetitive neuromodulation. There were no other significant differences (all $P$ values $>0.05$ ), with the exception of a lower startle magnitude in the neutral category after verum stimulation of the dmPFC $\left(t_{(38)}=-2.55, P=0.02\right.$, Cohen's $\left.d=0.83\right)$. In conclusion, inhibition of the left dIPFC (and to a lesser extent also the $\mathrm{dmPFC}$ ) attenuated the emotional modulation of the startle response particularly for positive stimuli.

Table 2 | Valence and arousal ratings in the dmPFC session

\begin{tabular}{|c|c|c|c|c|}
\hline & Verum $(n=20)$ Mean (SD) & Sham $(n=20)$ Mean (SD) & $t$ & $P$ \\
\hline Negative pre & $2.27(0.52)$ & $2.16(0.59)$ & -0.58 & 0.57 \\
\hline Neutral pre & $5.07(0.32)$ & $5.15(0.44)$ & 0.61 & 0.54 \\
\hline Neutral post & $5.06(0.24)$ & $5.15(0.46)$ & 0.81 & 0.43 \\
\hline Positive pre & $6.88(0.57)$ & $7.06(0.75)$ & 0.86 & 0.40 \\
\hline Positive post & $6.84(0.51)$ & $7.01(0.76)$ & 0.85 & 0.40 \\
\hline Negative post & $6.17(1.23)$ & $6.30(1.11)$ & 0.35 & 0.73 \\
\hline Neutral pre & $2.86(0.92)$ & $3.18(1.19)$ & 0.97 & 0.34 \\
\hline Neutral post & $2.98(1.08)$ & $3.35(1.30)$ & 0.98 & 0.34 \\
\hline Positive pre & $5.61(1.04)$ & $5.64(1.41)$ & 0.09 & 0.93 \\
\hline Positive post & $5.65(1.12)$ & $5.86(1.50)$ & 0.51 & 0.61 \\
\hline
\end{tabular}




\section{Discussion}

The rationale of the present study was to test whether fMRI-guided functional lesions of prefrontal cortex subregions would interfere with a pleasure-induced startle response suppression in healthy subjects. Our pre-TBS fMRI results revealed that the arousal assessment of stimuli subsequently used as emotional primers in the startle task evoked robust left-hemispheric responses in both dlPFC and dmPFC. Under sham stimulation, we observed the expected emotion modulation of the startle response, with negative foreground stimuli potentiating and positive ones reducing the startle magnitude. This pattern of results has been interpreted as evidence for motivational priming ${ }^{1}$. According to this view, aversive pictures may prime the defensive motivational system and subsequently lead to stronger protective responses including a facilitation of the startle reflex. Likewise, pleasant stimuli may engage the appetitive motivational system and therefore inhibit defensive responses. Our results show that inhibitory TBS of both target regions selectively diminished an appetitive modulation of the startle magnitude in the absence of changes in mood or cognitive emotion judgments.

Thus, to some extent, these findings mimic the aberrant emotional startle response profile exhibited by MDD patients, suggesting that inhibitory TBS over prefrontal cortex subregions can model distinct psychophysical abnormalities of the depressive phenotype in the healthy. Consistent with current perspectives that depression is characterized by an emotional context insensitivity ${ }^{32}$, a blunted startle response modulation has been reported for MDD patients $^{2,33-36}$ (but see Ref. 37) as well as for patients with a current anxiety disorder and a co-morbid depressive episode ${ }^{38}$. This aspect is reflected in our findings of a reduced frequency of both appetitive and aversive neuromodulation following focal disruption of the left dlPFC but not of the adjacent dmPFC, underscoring a pivotal role of the former in integrating bottom-up signals of emotional arousal. This valence-independent but focally restricted effect of inhibitory TBS on the arousal-related penetrance of emotion-startle interactions contrasts sharply with the observed deficit in startle magnitude modulation by hedonic stimuli - a valence-specific effect that occurred in response to inhibition of either the left dlPFC or $\mathrm{dmPFC}$ and cannot be explained by a disturbed integration of ascending arousal signals. Intriguingly, however, disruption of the left dlPFC has not only been shown to produce circumscribed cortical effects by inducing local decreases in metabolic activity ${ }^{39}$ but it also exerts more distant subcortical effects extending to the striatal reward system ${ }^{25-27,40}$, which may account for our findings.

Accumulating evidence implicates the left dlPFC - alone or in concert with adjacent medial regions ${ }^{41}$ - in representing motivational value in top-down control processes ${ }^{42}$. This is consistent with studies showing that excitation of the left dlPFC facilitates memory retrieval of positively valenced information ${ }^{43}$. While from a mechanistic perspective a TBS-induced focal dysfunction of the left dlPFC may be entirely sufficient to reduce an appetitive startle modulation, there is, however, substantial support for the assumption that inhibition of this target region is not limited to the cortex per se but propagates to frontostriatal neurocircuitry, thus compromising striatal responses to hedonic stimuli. For instance, animal lesion studies have shown that functional integrity of the striatum is essential for enabling a pleasure-induced down-regulation of the startle respons $\mathrm{e}^{44}$, and multiple lines of evidence converge on suggesting that dlPFC signals control striatal activity in a top-down regulative manner, thus adjusting value assignment to hedonic states and contexts ${ }^{31}$. Further supportive evidence comes from pharmacological challenges showing that the dopaminergic antagonist haloperidol blocks the otherwise robust startle reflex attenuation by dark-to-light transitions in rodents ${ }^{45}$, whereas in humans, homozygosity for the less active catechol-O-methyltransferase (COMT) gene variant, which confers increased striatal dopamine availability, is associated with an increased startle response inhibition by hedonic stimuli ${ }^{46}$.
In the present study, inhibitory TBS over the left dlPFC attenuated an appetitive startle modulation as did disruption of the left dmPFC, suggesting the latter might be an additional treatment target for antidepressant excitatory TMS (see also Ref. 47). From a clinical perspective, patients with $\mathrm{dmPFC}$ lesions are indeed at high risk for developing severe depression compared with other brain injury groups $^{48}$. Consistent with this increased vulnerability, a meta-analysis of voxel-based morphometry studies has confirmed extensive dmPFC gray matter deficits in MDD patients ${ }^{49}$. Intriguingly, a recent clinical trial involving TRD patients revealed marked symptom improvements in a subsample of patients with preserved hedonic function after TMS of the left $\mathrm{dmPFC}^{50}$. Our findings not only propose the dmPFC as a promising target for clinical studies, but also strongly inform the potential of TBS as a robust treatment regimen. The much shorter duration of TBS compared to classic TMS protocols may be a particular advantage and lead to improved rates of therapeutic adherence. A recent randomized sham-controlled study demonstrated that active theta-burst stimulation is a well-tolerated form of TMS and has promising antidepressant efficacy, particularly in depressed subjects within a certain range of treatment refractoriness ${ }^{51}$.

The observed dissociation of modulatory effects between tasks, with inhibitory TBS interfering with the influence of emotion on the startle response but not altering the cognitive evaluation of emotion, resembles the pattern of findings often observed in studies of emotional reactivity in MDD patients using the emotion-modulated startle paradigm ${ }^{2,33-36}$, as well as results from experiments using pharmacological probes ${ }^{52}$. This obvious disparity prompted some authors to suggest that aberrant emotion-startle interactions may be a much more sensitive psychophysical index of the emotional response deficits linked to the core pathophysiology of depression than cognitive self-report ratings ${ }^{36}$. Our results also resonate well with studies identifying distinct neural circuitries subserving the influence of emotion on the startle response and the cognitive evaluation of emotion ${ }^{53}$. The observed lack of inhibitory TBS effects on cognitive emotion ratings cannot be attributed to the temporal kinetics of TBS functional after-effects since these judgments preceded the startle paradigm and the prolonged intermittent TBS protocol used in our study has been shown to reliably produce depression-like plasticity for at least 60 minutes $^{30}$. Surprisingly, disruption of the dmPFC, but not the dlPFC, also led to a significantly reduced startle magnitude during the presentation of neutral foreground stimuli. Since inhibitory TBS had no effect on the baseline startle response per se, the most plausible explanation for this finding is an emotional shift induced by dmPFC inhibition.

Regarding potential limitations of our study, we note that only male volunteers were included, which may limit the generalizability of our findings to both sexes. Likewise, it has been demonstrated that the modulatory influence of emotional stimuli on the startle reflex may vary as a function of the participants' age, with older adults potentially exhibiting altered emotion-startle interactions $^{54}$. Thus, the effects of inhibitory TBS as documented by the present study cannot necessarily be extended to older populations. In addition, experimental TMS effects are often moderated by state anxiety or $\operatorname{mood}^{55}$. However, we can rule out any nonspecific contributions from these parameters, since there were neither pretreatment nor post-treatment differences in state anxiety or mood between the sham and verum treated group (cf. Supplemental Tables S3 and S4). Furthermore, consistent with other TMS studies targeting the left dlPFC ${ }^{56,57}$, we observed no discrepancies in attentional performance. Moreover, we controlled for possible placebo effects by using a placebo coil that produced a slight sensory stimulation. Related to this, we note that subjects were unaware of whether they had received verum or sham treatment, and pleasantness ratings also indicate no between-group differences (cf. Supplemental Information). 
In conclusion, we here provide the first evidence that fMRI-guided disruption of the left dlPFC or dmPFC in healthy subjects can induce malfunctioning of the appetitive motivational system, evident in a diminished suppression of the startle magnitude by hedonic stimuli. Our findings thus suggest that prefrontal functional integrity is critical for mediating a down-regulation of avoidance responses by pleasure signals, thus protecting the brain from a depressogenic preponderance of defensive stress.

\section{Methods}

Participants. Forty-one healthy, non-smoking, heterosexual, male adults (mean age $\pm \mathrm{SD}=24.12 \pm 3.66$ years) participated in the present study. All subjects were recruited from the University of Bonn and gave written informed consent, which was approved by the Institutional Review Board (IRB) of the Medical Faculty of the University of Bonn. All experimental protocols and procedures were conducted in accordance with the IRB guidelines for experimental testing and were in compliance with the latest revision of the Declaration of Helsinki.

Subjects displaying fewer than $50 \%$ satisfactory blink responses in the startle paradigm (verum group, $\mathrm{n}=1$ ) were excluded. All remaining participants (verum group: $\mathrm{n}=20$, mean age $\pm \mathrm{SD}=23.85 \pm 3.25$ years; sham group: $\mathrm{n}=20,24.45 \pm$ 4.16 years) showed normal cognitive performance and were free of current and past physical or psychiatric illness, as assessed by medical history and a Structured Clinical Interview for DSM-IV axis I (SCID-I) and axis II disorders (SCID-II). Notably, there were no a priori differences between the verum and sham treated groups regarding age, education, and pre-treatment neuropsychological parameters (all $P$ values $>$ 0.05; for details see Supplemental Table S1).

Experimental tasks. $f M R I$ localizer task. To identify the anatomical target regions for subsequent inhibitory TBS, all participants initially underwent fMRI scanning on a functional localizer task requiring them to rate the emotional intensity (arousal) of negative, neutral and positive pictures selected from one of two similar stimulus sets (A and B). Neural responses associated with this arousal-based evaluation (contrast: negative and positive $>$ neutral) were analyzed using BrainVoyager $\mathrm{QX}^{58}$. Data were acquired with a 1.5 Tesla Siemens Magnetom Espree MRI system (Siemens, Erlangen, Germany). Details on the task, fMRI procedure and analyses are reported in the Supplemental Information section. After fMRI scanning, subjects were randomly assigned to either verum or sham TBS, with the first of two subsequent TBS sessions commencing three months after fMRI data acquisition.

Cognitive emotion judgment task. Immediately after each TBS session, subjects were exposed to a cognitive emotion judgment task. Specifically, they used a 9-point selfassessment manikin (SAM) scale ${ }^{59}$ to rate the arousal $(1$, calm; 9 , excited) and valence (1, negative; 9 , positive) of picture set A or B. Each picture set contained 30 negative, 30 neutral and 30 positive stimuli carefully selected from the International Affective Picture System (IAPS) ${ }^{59}$. The order of picture sets was balanced, i.e. if set A was shown before the TBS procedure, the post-TBS rating was completed using set B. Subsequently, all subjects were submitted to the startle response task.

Emotion-modulated startle paradigm. During this task, participants were exposed to acoustic startle probes presented either alone or paired with a picture. The paradigm featured 20 negative, 20 neutral and 20 positive pictures of the same picture sets (A and B) used in the fMRI task three months before. The pictures were presented for $5 \mathrm{~s}$ and were shown in a pseudo-randomized order. The startle stimulus consisted of a single 50 -ms burst of white noise $(100 \mathrm{~dB})$ with nearly instantaneous rise and was delivered binaurally via headphones during $60 \%$ of the pictures (i.e. 12 from each category) at 2-4 s after picture onset. A $70-\mathrm{dB}$ white noise background was present throughout the experiment. Facial electromyographic (EMG) activity was recorded from two $\mathrm{Ag} / \mathrm{AgCl}$ electrodes placed over the orbicularis oculi muscle below the left eye $^{60}$. A ground electrode was placed behind the subjects' left ear. A commercial system (Contact Precision Instruments, Cambridge, MA) was used for stimulus delivery and psychophysiological recordings. In addition, 18 of 59 interstimulus intervals were accompanied by startle probes to reduce predictability. To account for early habituation, the experiment started with the presentation of five startle probes in 2-s intervals with no picture and five startle probes during the presentation of a neutral picture. The facial EMG signal was digitized at a rate of $1000 \mathrm{~Hz}$ and amplified with a high-pass filter of $30 \mathrm{~Hz}$ and a low-pass filter of $500 \mathrm{~Hz}$. EMG data were rectified and smoothed by a 4-point moving average. Startle eyeblink reflex was calculated as the difference between the maximum increase of EMG activity in a time interval between 20 and 100 ms after startle probe onset and the mean EMG of the 50$\mathrm{ms}$ baseline directly preceding the onset. All EMG data were z-transformed withinsubject and then converted into $T$-scores to reduce between-subjects variability and skew. The frequency of emotion modulation was calculated as the proportion of $z$ values smaller than zero (positive category) or larger than zero (negative category) relative to the number of all valid startle trials (all startle trials minus artifacts). The EMG recordings were visually inspected, and trials with excessive noise were excluded from further analysis (overall $9 \%$ of all trials). Trials with no perceptible eyeblink reflex were assigned a magnitude of zero and included in the analysis (overall $8 \%$ of all trials). Subjects displaying fewer than $50 \%$ satisfactory blink responses in the paradigm (verum group, $\mathrm{n}=1$ ) were excluded.
Theta-burst TMS protocol. We applied a randomized, placebo-controlled, betweengroup (sham vs. verum TBS) design, with all participants undergoing two sessions of TBS, i.e. one with the dlPFC and another one with the dmPFC as target region, in a balanced order. Assignment of the target region was balanced with respect to treatment (sham vs. verum TMS) and picture set (A vs. B). TBS was applied using a Magstim Super Rapid 2 (The Magstim Company Ltd, Whitland, U.K.) and a figureof-eight TMS coil (air film double $70 \mathrm{~mm}$ coil). For the sham treatment, a placebo coil (double $70 \mathrm{~mm}$ ) was used that provides slight sensory stimulation and discharge noise, however, without stimulating cortical tissue. We administered a prolonged intermittent theta-burst protocol which consisted of bursts containing 3 pulses at $50 \mathrm{~Hz}$ repeated at $5 \mathrm{~Hz}$. The protocol lasted $390 \mathrm{~s}$ (40 cycles with a total of 1200 pulses). This protocol has been previously shown to produce long-lasting inhibitory effects $^{30,61}$. Stimulation intensity was set at $80 \%$ of the individual active motor threshold (mean active motor threshold: $37.4 \%$ of maximum stimulator output, minimum $31 \%$, maximum 53\%).

A frameless stereotactic system (BrainVoyager TMS Neuronavigator system; Brain-Innovation, Maastricht, The Netherlands) was used to ensure precise coil positioning ${ }^{62}$. The target sites were determined as the Talairach coordinates of those areas in the fMRI group analysis which exhibited the most robust activation for the contrast [Emotional $>$ Neutral] (dlPFC: $-40,28,24$; dmPFC: $-1,52,33)$. These normalized stereotaxic coordinates were back-transformed to the individual subject's brain coordinates in native space by reversing the native-to-Talairach transformation procedure. Then, TMS fMRI guidance was based on data in AC-PC space (rotating the cerebrum into the anterior commissure - posterior commissure plane). The coil positioning was supported by a rack and the coil was held tangentially to the skull with the coil handle oriented perpendicular to the middle (dlPFC) or superior (dmPFC) frontal gyrus. The distance between the center of the coil and target point was kept as small as possible.

1. Lang, P. J., Bradley, M. M. \& Cuthbert, B. N. Emotion, motivation, and anxiety: brain mechanisms and psychophysiology. Biol. Psychiatry 44, 1248-1263 (1998)

2. Kaviani, H. et al. Affective modulation of the startle response in depression: influence of the severity of depression, anhedonia, and anxiety. J. Affect. Disord. 83, 21-31 (2004).

3. Kendler, K. S., Karkowski, L. M. \& Prescott, C. A. Causal relationship between stressful life events and the onset of major depression. Am. J. Psychiatry 156, 837-841 (1999).

4. Belmaker, R. H. \& Agam, G. Major depressive disorder. N. Engl. J. Med. 358 , 55-68 (2008).

5. Krishnan, V. \& Nestler, E. J. The molecular neurobiology of depression. Nature 455, 894-902 (2008).

6. Pizzagalli, D. A., Jahn, A. L. \& O'Shea, J. P. Toward an objective characterization of an anhedonic phenotype: a signal-detection approach. Biol. Psychiatry 57, 319-327 (2005)

7. Trew, J. L. Exploring the roles of approach and avoidance in depression: an integrative model. Clin. Psychol. Rev. 31, 1156-1168 (2011).

8. Mathers, C. D. \& Loncar, D. Projections of global mortality and burden of disease from 2002 to 2030. PLoS Med. 3, e442 (2006).

9. Lefaucheur, J. P. et al. Evidence-based guidelines on the therapeutic use of repetitive transcranial magnetic stimulation (rTMS). Clin. Neurophysiol. DOI: 10.1016/j.clinph.2014.05.021 (2014).

10. Berlim, M. T., van den Eynde, F., Tovar-Perdomo, S. \& Daskalakis, Z. J. Response, remission and drop-out rates following high-frequency repetitive transcranial magnetic stimulation (rTMS) for treating major depression: a systematic review and meta-analysis of randomized, double-blind and sham-controlled trials. Psychol. Med. 44, 225-239 (2014).

11. Figner, B. et al. Lateral prefrontal cortex and self-control in intertemporal choice. Nat. Neurosci. 13, 538-539 (2010).

12. Ott, D. V., Ullsperger, M., Jocham, G., Neumann, J. \& Klein, T. A. Continuous theta-burst stimulation (cTBS) over the lateral prefrontal cortex alters reinforcement learning bias. Neuroimage 57, 617-623 (2011).

13. Ahn, H. M., Kim, S. E. \& Kim, S. H. The effects of high-frequency rTMS over the left dorsolateral prefrontal cortex on reward responsiveness. Brain Stimul. 6, 310-314 (2013)

14. Perach-Barzilay, N. et al. Asymmetry in the dorsolateral prefrontal cortex and aggressive behavior: a continuous theta-burst magnetic stimulation study. Social Neuroscience 8, 178-188 (2013)

15. Knoch, D., Schneider, F., Schunk, D., Hohmann, M. \& Fehr, E. Disrupting the prefrontal cortex diminishes the human ability to build a good reputation. Proc. Natl. Acad. Sci. U. S. A. 106, 20895-20899 (2009).

16. Knoch, D. et al. Disruption of right prefrontal cortex by low-frequency repetitive transcranial magnetic stimulation induces risk-taking behavior. J. Neurosci. 26, 6469-6472 (2006).

17. Cho, S. S. et al. Continuous theta burst stimulation of right dorsolateral prefrontal cortex induces changes in impulsivity level. Brain Stimul. 3, 170-176 (2010).

18. Tassy, S. et al. Disrupting the right prefrontal cortex alters moral judgement. Soc. Cogn. Affect. Neurosci. 7, 282-288 (2012).

19. George, M. S. et al. Changes in mood and hormone levels after rapid-rate transcranial magnetic stimulation (rTMS) of the prefrontal cortex. J. Neuropsychiatry Clin. Neurosci. 8, 172-180 (1996). 
20. Pascual-Leone, A., Catala, M. D. \& Pascual-Leone Pascual, A. Lateralized effect of rapid-rate transcranial magnetic stimulation of the prefrontal cortex on mood. Neurology 46, 499-502 (1996).

21. Mosimann, U. P., Rihs, T. A., Engeler, J., Fisch, H. \& Schlaepfer, T. E. Mood effects of repetitive transcranial magnetic stimulation of left prefrontal cortex in healthy volunteers. Psychiatry Res. 94, 251-256 (2000).

22. Baeken, C., Leyman, L., De Raedt, R., Vanderhasselt, M. A. \& D’Haenen, H. Left and right High Frequency repetitive Transcranial Magnetic Stimulation of the dorsolateral prefrontal cortex does not affect mood in female volunteers. Clin. Neurophysiol. 119, 568-575 (2008).

23. Schaller, G. et al. Repetitive transcranial magnetic stimulation influences mood in healthy male volunteers. J. Psychiatr. Res. 45, 1178-1183 (2011).

24. Baeken, C. et al. Right prefrontal HF-rTMS attenuates right amygdala processing of negatively valenced emotional stimuli in healthy females. Behav. Brain Res. 214, 450-455 (2010).

25. Strafella, A. P., Paus, T., Barrett, J. \& Dagher, A. Repetitive transcranial magnetic stimulation of the human prefrontal cortex induces dopamine release in the caudate nucleus. J. Neurosci. 21, RC157 (2001).

26. Pogarell, O. et al. Acute prefrontal rTMS increases striatal dopamine to a similar degree as D-amphetamine. Psychiatry Res. 156, 251-255 (2007).

27. Ko, J. H. et al. Theta burst stimulation-induced inhibition of dorsolatera prefrontal cortex reveals hemispheric asymmetry in striatal dopamine release during a set-shifting task: a TMS-[(11)C]raclopride PET study. Eur. J. Neurosci. 28, 2147-2155 (2008).

28. Salamone, J. D. \& Correa, M. The mysterious motivational functions of mesolimbic dopamine. Neuron 76, 470-485 (2012).

29. Grimm, S. et al. Imbalance between left and right dorsolateral prefrontal cortex in major depression is linked to negative emotional judgment: an fMRI study in severe major depressive disorder. Biol. Psychiatry 63, 369-376 (2008).

30. Gamboa, O. L., Antal, A., Moliadze, V. \& Paulus, W. Simply longer is not better: reversal of theta burst after-effect with prolonged stimulation. Exp. Brain Res. 204, 181-187 (2010).

31. Aarts, E., van Holstein, M. \& Cools, R. Striatal Dopamine and the Interface between Motivation and Cognition. Front. Psychol. 2, 163 (2011).

32. Rottenberg, J., Gross, J. J. \& Gotlib, I. H. Emotion context insensitivity in major depressive disorder. J. Abnorm. Psychol. 114, 627-639 (2005).

33. Allen, N. B., Trinder, J. \& Brennan, C. Affective startle modulation in clinical depression: preliminary findings. Biol. Psychiatry 46, 542-550 (1999).

34. Dichter, G. S., Tomarken, A. J., Shelton, R. C. \& Sutton, S. K. Early- and late-onset startle modulation in unipolar depression. Psychophysiology 41, 433-440 (2004).

35. Forbes, E. E., Miller, A., Cohn, J. F., Fox, N. A. \& Kovacs, M. Affect-modulated startle in adults with childhood-onset depression: relations to bipolar course and number of lifetime depressive episodes. Psychiatry Res. 134, 11-25 (2005).

36. Dichter, G. S. \& Tomarken, A. J. The chronometry of affective startle modulation in unipolar depression. J. Abnorm. Psychol. 117, 1-15 (2008).

37. Grillon, C., Franco-Chaves, J. A., Mateus, C. F., Ionescu, D. F. \& Zarate, C. A. Major depression is not associated with blunting of aversive responses; evidence for enhanced anxious anticipation. PloS one 8, e70969 (2013).

38. Taylor-Clift, A., Morris, B. H., Rottenberg, J. \& Kovacs, M. Emotion-modulated startle in anxiety disorders is blunted by co-morbid depressive episodes. Psychol. Med. 41, 129-139 (2011).

39. Tupak, S. V. et al. Inhibitory transcranial magnetic theta burst stimulation attenuates prefrontal cortex oxygenation. Hum. Brain Mapp. 34, 150-157 (2013).

40. Pogarell, O. et al. Striatal dopamine release after prefrontal repetitive transcranial magnetic stimulation in major depression: preliminary results of a dynamic [123I] IBZM SPECT study. J. Psychiatr. Res. 40, 307-314 (2006).

41. Kouneiher, F., Charron, S. \& Koechlin, E. Motivation and cognitive control in the human prefrontal cortex. Nat. Neurosci. 12, 939-945 (2009).

42. Jimura, K., Locke, H. S. \& Braver, T. S. Prefrontal cortex mediation of cognitive enhancement in rewarding motivational contexts. Proc. Natl. Acad. Sci. U. S. A. 107, 8871-8876 (2010)

43. Balconi, M. \& Ferrari, C. Left DLPFC rTMS stimulation reduced the anxiety bias effect or how to restore the positive memory processing in high-anxiety subjects. Psychiatry Res. 209, 554-559 (2013).

44. Koch, M., Schmid, A. \& Schnitzler, H. U. Pleasure-attenuation of startle is disrupted by lesions of the nucleus accumbens. Neuroreport 7, 1442-1446 (1996).

45. Schmajuk, N. A., Larrauri, J. A., De la Casa, L. G. \& Levin, E. D. Attenuation of auditory startle and prepulse inhibition by unexpected changes in ambient illumination through dopaminergic mechanisms. Behav. Brain Res. 197, 251-261 (2009).

46. Klauke, B. et al. Affect-modulated startle: interactive influence of catechol-Omethyltransferase Val158Met genotype and childhood trauma. PloS one 7, e39709 (2012).
47. Downar, J. \& Daskalakis, Z. J. New targets for rTMS in depression: a review of convergent evidence. Brain Stimul. 6, 231-240 (2013).

48. Koenigs, M. et al. Distinct regions of prefrontal cortex mediate resistance and vulnerability to depression. J. Neurosci. 28, 12341-12348 (2008).

49. Bora, E., Fornito, A., Pantelis, C. \& Yucel, M. Gray matter abnormalities in Major Depressive Disorder: a meta-analysis of voxel based morphometry studies. J. Affect. Disord. 138, 9-18 (2012).

50. Downar, J. et al. Anhedonia and Reward-Circuit Connectivity Distinguish Nonresponders from Responders to Dorsomedial Prefrontal Repetitive Transcranial Magnetic Stimulation in Major Depression. Biol. Psychiatry (2013).

51. Li, C. T. et al. Efficacy of prefrontal theta-burst stimulation in refractory depression: a randomized sham-controlled study. Brain 137, 2088-2098 (2014).

52. Striepens, N. et al. Oxytocin facilitates protective responses to aversive social stimuli in males. Proc. Natl. Acad. Sci. U. S. A. 109, 18144-18149 (2012).

53. Anders, S., Lotze, M., Erb, M., Grodd, W. \& Birbaumer, N. Brain activity underlying emotional valence and arousal: a response-related fMRI study. Hum. Brain Mapp. 23, 200-209 (2004).

54. Feng, M. C., Courtney, C. G., Mather, M., Dawson, M. E. \& Davison, G. C. Agerelated affective modulation of the startle eyeblink response: older adults startle most when viewing positive pictures. Psychol. Aging 26, 752-760 (2011).

55. Baeken, C., Vanderhasselt, M. A. \& De Raedt, R. Baseline 'state anxiety' influences HPA-axis sensitivity to one sham-controlled HF-rTMS session applied to the right dorsolateral prefrontal cortex. Psychoneuroendocrinology 36, 60-67 (2011).

56. Rounis, E. et al. Acute changes in frontoparietal activity after repetitive transcranial magnetic stimulation over the dorsolateral prefrontal cortex in a cued reaction time task. J. Neurosci. 26, 9629-9638 (2006).

57. Leyman, L., De Raedt, R., Vanderhasselt, M. A. \& Baeken, C. Influence of high frequency repetitive transcranial magnetic stimulation over the dorsolateral prefrontal cortex on the inhibition of emotional information in healthy volunteers. Psychol. Med. 39, 1019-1028 (2009).

58. Goebel, R. BrainVoyager - past, present, future. Neuroimage 62, 748-756 (2012).

59. Lang, P. J., Bradley, M. M. \& Cuthbert, B. N. International affective picture system (IAPS): Affective ratings of pictures and instruction manual. Technical Report A-6. (Gainesville, FL: University of Florida, 2005).

60. Fridlund, A. J. \& Cacioppo, J. T. Guidelines for human electromyographic research. Psychophysiology 23, 567-589 (1986).

61. Huang, Y. Z., Edwards, M. J., Rounis, E., Bhatia, K. P. \& Rothwell, J. C. Theta burst stimulation of the human motor cortex. Neuron 45, 201-206 (2005).

62. Sack, A. T. et al. Optimizing functional accuracy of TMS in cognitive studies: a comparison of methods. J. Cogn. Neurosci. 21, 207-221 (2009).

\section{Acknowledgments}

R.H. was supported by a Starting Independent Researcher Grant ('NEMO -

Neuromodulation of Emotion') jointly provided by the Ministry of Innovation, Science, Research \& Technology of the German State of North Rhine-Westphalia (MIWFT) and the University of Bonn.

\section{Author contributions}

R.H. and D.S. designed the experiments; S.A. and D.S. conducted the experiments; R.H. and D.S. analyzed the data; J.R. contributed new reagents/analytic tools; R.H., S.A., T.E.S., W.M. and D.S. wrote the paper. All authors read and approved the manuscript in its current form.

\section{Additional information}

Supplementary information accompanies this paper at http://www.nature.com/ scientificreports

Competing financial interests: The authors declare no competing financial interests. How to cite this article: Hurlemann, R. et al. Diminished appetitive startle modulation following targeted inhibition of prefrontal cortex. Sci. Rep. 5, 8954; DOI:10.1038/srep08954 (2015).

This work is licensed under a Creative Commons Attribution 4.0 International License. The images or other third party material in this article are included in the article's Creative Commons license, unless indicated otherwise in the credit line; if the material is not included under the Creative Commons license, users will need to obtain permission from the license holder in order to reproduce the material. To view a copy of this license, visit http://creativecommons.org/licenses/by/4.0/ 